\title{
Spin Relaxation in Germanium Nanowires
}

\author{
Ashish Kumar, M. W. Akram, and Bahniman Ghosh \\ Department of Electrical Engineering, Indian Institute of Technology, Kanpur 208016, India \\ Correspondence should be addressed to Bahniman Ghosh, bahniman@iitk.ac.in
}

Received 2 April 2012; Accepted 6 May 2012

Academic Editors: M. Bescond and J.-M. Shen

Copyright ( 12012 Ashish Kumar et al. This is an open access article distributed under the Creative Commons Attribution License, which permits unrestricted use, distribution, and reproduction in any medium, provided the original work is properly cited.

\begin{abstract}
We use semiclassical Monte Carlo approach along with spin density matrix calculations to model spin polarized electron transport. The model is applied to germanium nanowires and germanium two-dimensional channels to study and compare spin relaxation between them. Spin dephasing in germanium occurs because of Rashba Spin Orbit Interaction (structural inversion asymmetry) which gives rise to the D'yakonov-Perel (DP) relaxation. In germanium spin flip scattering due to the Elliot-Yafet (EY) mechanism also leads to spin relaxation. The spin relaxation tests for both $1 \mathrm{D}$ and $2 \mathrm{D}$ channels are carried out at different values of temperature and driving electric field, and the variation in spin relaxation length is recorded. Spin relaxation length in a nanowire is found to be much higher than that in a $2 \mathrm{D}$ channel due to suppression of DP relaxation in a nanowire. At lower temperatures the spin relaxation length increases. This suggests that spin relaxation in germanium occurs slowly in a $1 \mathrm{D}$ channel (nanowires) and at lower temperatures. The electric field dependence of spin relaxation length was found to be very weak.
\end{abstract}

\section{Introduction}

Of late, intensive experimental and theoretical studies have been conducted on the physics of electron spins due to the enormous promise displayed by the spin-based devices [1]. Spin transport in semiconductors has been continuously investigated due to the possibility of integration of spintronics with semiconductor technology. This integration has attracted huge research interest due to its prospects [2-5] in implementing novel devices that can operate at much less power levels and higher processing speeds. Spintronicsbased devices thus promise highly improved performance over their contemporary electronic counterparts. The suitability of present semiconductor materials in spintronicbased applications needs to be established to bring about successful integration of the two and to be able to achieve the advantages listed above.

The basic idea of the spintronic-based devices is to use the spin degree of freedom. At the source, information is encoded as spin state of individual electrons and is then injected into the material. During its motion in the material, the electrons undergo scattering and hence the electron spin states relax or depolarize as they move in the channel. This is the process of spin relaxation. Spin detection is done at the drain. Our paper here deals with the second process of spin relaxation in a material. Spin relaxation lengths or spin dephasing lengths represent the distance from the source in which the spin polarization of an ensemble of electrons gets randomized and thus loses the information. We do not want the electrons to lose encoded information before the operation is complete. Thus information regarding spin relaxation lengths is critical to realize any useful spintronicbased device.

Intensive theoretical and experimental research has been conducted to study spin relaxation in metals and semiconductors. Several III-V and II-VI materials have been studied to ascertain their spin properties [5-9]. In [7] spin transport is studied experimentally in GaAs. In [8] spin-polarized transport in GaAs/GaAlAs quantum wells is investigated using Monte Carlo simulations. Spin relaxation in silicon $[10,11]$ has also been studied experimentally. Spin dephasing has also been studied at different conditions of temperature, applied electric field, and for different dimensionality of systems $[12,13]$ by researchers in a bid to find the optimum conditions of use of such materials in devices. In [12] spin transport in GaAs nanowires is modeled at different temperatures and different driving electric fields. Comparison of spin-polarized transport in 1D and 2D III-V heterostructures was done in [13]. 
Though silicon has been the workhorse of the semiconductor materials since long, germanium has some superior properties [14] to silicon and thus is a material of interest. Germanium has a smaller indirect bandgap of $0.66 \mathrm{eV}$ while silicon has an indirect bandgap of $1.12 \mathrm{eV}$. Germanium has higher electron and hole mobility than silicon. Also germanium has a much lower resistivity than silicon, and thus germanium offers great opportunities for device scaling especially when it comes to low drive voltage and high drive current. Recently Ge nanowires have attracted attention due to their possible role in future nanoscale devices such as light-emitting diodes (LEDs), logic gates, nanoscale sensors, and so forth. The growing popularity of germanium thus requires us to assess their performance as a spintronic material. However theoretical work on spin relaxation in germanium is still in its very early stages. Much of the work on germanium till now has been experimental [15-17]. This growing importance of germanium coupled with the fact that Monte Carlo simulations have not yet been used to study spin-dephasing lengths in germanium (to the best of our knowledge) motivates us to take such a study on germanium.

Spin relaxation in semiconductors can occur via different spin relaxation mechanisms, such as D'yakonov-Perel (DP) [18] mechanism, Bir-Aronov-Pikus [19] mechanism, and Elliott-Yafet (EY) [20] mechanism. Bir-Aronov-Pikus mechanism is present in p-type semiconductors only and is hence not relevant in our study. In germanium DP mechanism is present. Also being a smaller bandgap material with a high spin orbit coupling $(=290 \mathrm{meV})$, EY mechanism is also a dominant spin-relaxing mechanism. DP relaxation is a continuous process that occurs even during the free flight time of electrons, and it leads to a continuous spin precession about the effective magnetic field. EY relaxation on the other hand is an instantaneous spin-flip scattering and is therefore a discrete process that occurs only during scattering. Therefore EY relaxation is treated as a scattering process.

In addition to the above studies, charge transport in nanowires has been modeled using the nonequilibrium Green's function technique [21]. Semiclassical Monte Carlo approach is used to model electron transport in $2 \mathrm{D}$ germanium channels and in 1D germanium nanowires. The Monte Carlo method [22-24] along with spin density matrix [24] is used to model spin transport of electrons in both 2D and 1D systems. Monte Carlo approach is used since it is able to update spin evolution dynamically in step with the momentum evolution due to electron transport. In conformity with some of the previous works [12, 13], improvement in spin relaxation on confinement is observed. Spin relaxation is reinvestigated at different temperatures and different driving electric fields. A similar study was done by us on silicon [25], and this paper is a followup of the earlier work.

The paper is organized as follows. In Section 2 we discuss the model used for our simulations. In Section 3 the parameters used in the simulations are mentioned. The results obtained are shown, and a discussion follows on the results in the Section 3. The conclusion is presented in Section 4.

\section{Model}

A detailed account of the Monte Carlo method [22-24] and spin transport model $[6,12,24,25]$ is presented elsewhere. Here we shall be discussing only the key features of the model and the essential modifications from our previous work [25]. The coordinate system is so chosen such that $x$ is along the length, $y$ is along the width, and $z$ is the along the thickness of the device. In the 2D system the electrons can move in the $x$ direction and the $y$ direction, while in the 1D system electrons are free to move only in the $x$ direction.

Germanium is an elemental semiconductor and possesses crystallographic inversion symmetry [15, 17]. As a result the Dresselhaus spin orbit interaction is absent $[15,17]$ in germanium. However a transverse electric field breaks the structural inversion symmetry. The structural inversion asymmetry thus present leads to the Rashba spin orbit interaction. The Rashba spin orbit coupling causes spin relaxation in the channel via the D'yakonov-Perel (DP) mechanism.

In the Monte Carlo method, transport is simulated by free flights occasionally disrupted by scattering events. The flow chart of the Monte Carlo method is shown in Figure 1 and is taken from [22].

During free flight, in which no scattering occurs, the temporal evolution of spin [25] is given by following equation:

$$
\frac{d \vec{S}}{d t}=\vec{\Omega} \times \vec{S}
$$

$\vec{\Omega}$, precession vector, has only the Rashba component which is given by

$$
\Omega_{R}\left(k_{x}\right)^{1 \mathrm{D}}=-\frac{2 \alpha k_{x} \hat{j}}{\hbar},
$$

where $\alpha$ is the Rashba coefficient [26], given by

$$
\alpha=\frac{\hbar^{2}}{2 m^{*}} \frac{\Delta}{E_{g}} \frac{2 E_{g}+\Delta}{\left(E_{g}+\Delta\right)\left(3 E_{g}+2 \Delta\right)} e E,
$$

where $\Delta$ is the spin orbit splitting, $e$ is the electron charge, $m^{*}$ is the effective mass, $E_{g}$ is the bandgap, and $E$ is the transverse electric field.

Using (2) in (1) and expressing spin vector as $\vec{S}=$ $S_{x} \hat{i}+S_{y} \hat{j}+S_{z} \hat{k}$, we arrive at the following relations for each component of spin;

$$
\begin{gathered}
\frac{d S_{x}}{d t}=-\frac{2}{\hbar} \alpha k_{x} S_{z}, \\
\frac{d S_{y}}{d t}=0, \\
\frac{d S_{z}}{d t}=\frac{2}{\hbar} \alpha k_{x} S_{x} .
\end{gathered}
$$

The conduction band of Ge [27] consists of the four lowest energy $\langle 111\rangle \mathrm{L}$ valleys at the edges of the Brillouin zone, the $\langle 000\rangle \Gamma$ valley at the zone center, and the six $\langle 100\rangle \Delta$ 


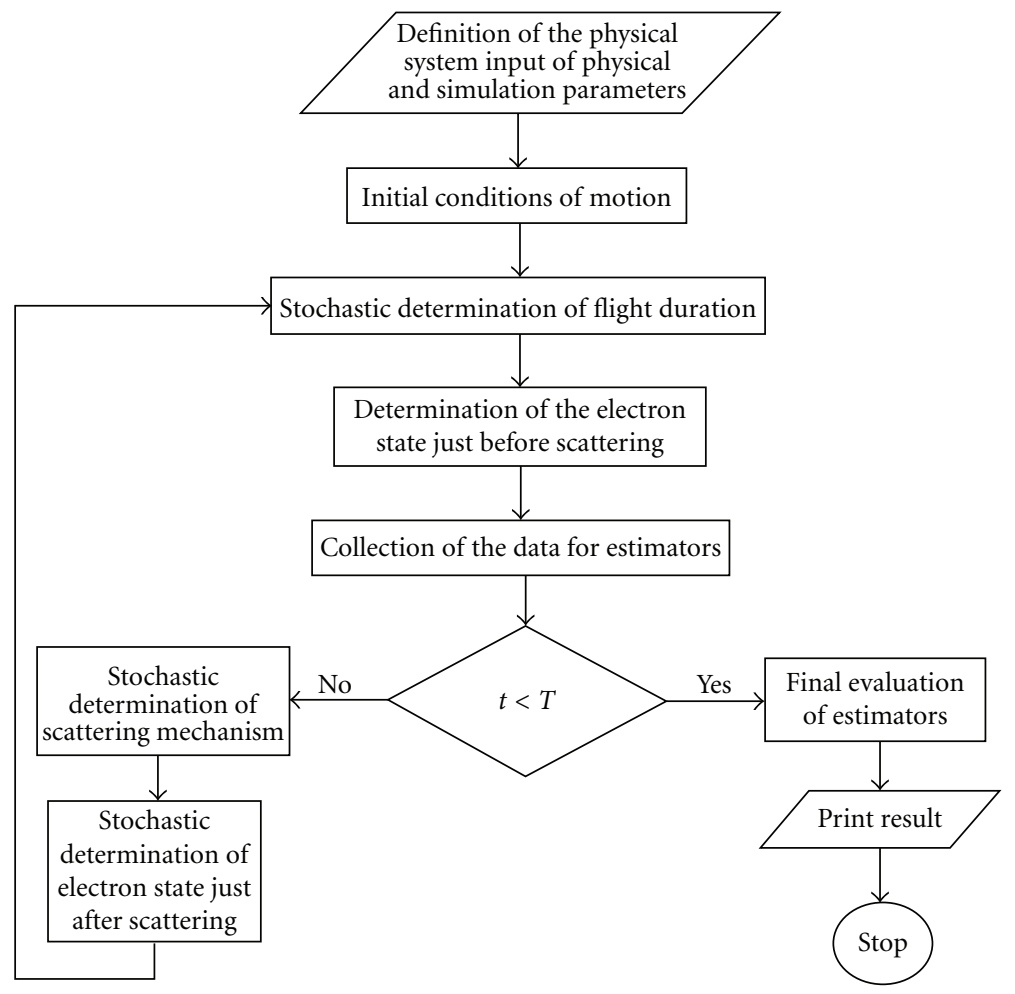

Figure 1: Flow chart of the Monte Carlo simulation [22].

valleys located near the zone edges. The $\langle 111\rangle$ minimum is $0.14 \mathrm{eV}$ below the $\langle 000\rangle$ minimum and $0.18 \mathrm{eV}$ below the $\langle 100\rangle$ minimum.

We consider here that since the $\mathrm{L}$ valleys are lower in energy than the other two valleys, majority of electrons are concentrated in these $\langle 111\rangle$ valleys. Thus we do not consider the other two valleys, that is, the $\Gamma$ and the $\Delta$ valleys for the sake of our simulation assuming that they are completely depopulated and hence have negligible effects on our final results. We would like to mention here that this assumption may be relaxed for a more rigorous treatment of Ge nanowires, since, it is known that confinement is expected to modify the energy of the other valleys (i.e., $\Gamma$ and $\Delta$ ) which can become the lowest in energy and therefore be also populated.

Also we assume here that the electric field applied is in the $\langle 100\rangle$ direction, and hence the four $\mathrm{L}$ valleys remain equivalent.

The scattering processes considered are intravalley and intervalley phonon scattering, surface roughness scattering, and ionized impurity scattering. While considering phonon scattering, both optical phonons and acoustic phonons have been taken into account. The electrons will not always remain in the lowest ground subband and will make transitions to the higher subbands. Therefore, subbands are included in the simulation, and intervalley and intravalley intersubband scatterings are thus accounted for.

Spin flip scattering [28] via the Elliot Yafet mechanism is accounted for in both the 1D and 2D systems. There occurs a finite probability for spin flip due to any perturbing potential even if the perturbation is spin independent (which might be present because of phonons, ionized impurities). The spin relaxation time is given by

$$
\frac{1}{\tau_{S}^{E Y}}=A\left(\frac{k_{B} T}{E_{g}}\right)^{2} \eta^{2}\left(\frac{1-\eta / 2}{1-\eta / 3}\right)^{2} \frac{1}{\tau_{p}},
$$

where $E_{g}$ is the bandgap, $\eta=\Delta /\left(E_{g}+\Delta\right)$ with $\Delta$ as the spin orbit splitting of the valence band, $\tau_{p}$ is the momentum relaxation time, and $A$ is a dimensionless constant and varies from 2 to 6 . We have chosen $A$ as 4 for our simulations.

The formula for scattering rates calculations in nanowire and 2D channels are taken from [29-35] and have been discussed in our work on silicon [25].

The 2D channel has $5 \mathrm{~nm}$ as the thickness and $125 \mathrm{~nm}$ as the width. The nanowire is taken to be of cross-section $5 \mathrm{~nm} \times 5 \mathrm{~nm}$. The doping density is taken to be $4 \times 10^{25} / \mathrm{m}^{3}$. The effective field is taken to be $100 \mathrm{kV} / \mathrm{cm}$ which is a reasonable value for germanium channels. This effective field acts as the transverse symmetry breaking field and leads to the Rashba spin orbit coupling.

Accounting for the confinement, four subbands are taken for the sake of simulation in each valley for both the channels. The moderate values of driving electric field $(100 \mathrm{~V} / \mathrm{cm}$ to $5 \mathrm{kV} / \mathrm{cm}$ ) used ensure that the majority of electrons are restricted to the first four subbands. Also due to very small $(5 \mathrm{~nm})$ transverse dimensions of the channels, the higher subbands will be too much higher up in energy to the effect that we can assume them to be depopulated. Similarly in [8] 3 subbands were considered for the purpose of 


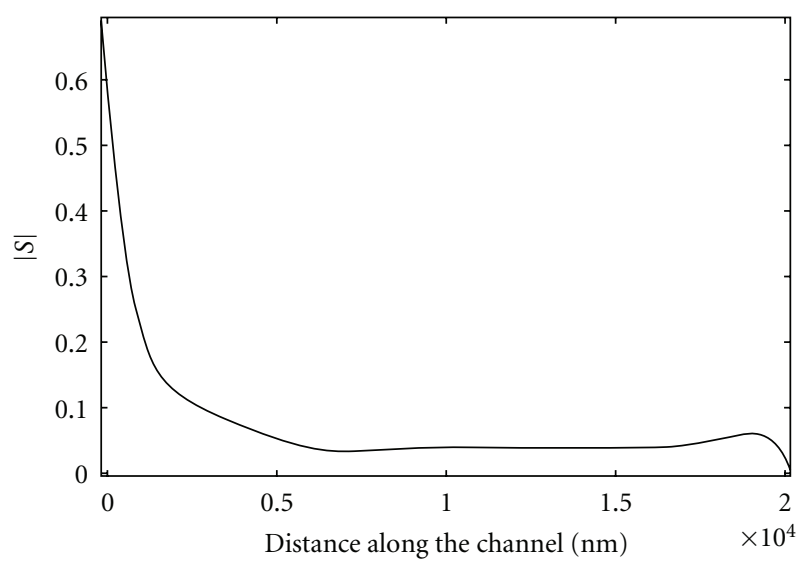

Figure 2: Variation of magnitude of spin along the channel of a germanium nanowire.

determining spin-dephasing lengths in a III-V compound. The energy levels of subbands are computed considering an infinite potential a well approximation. The other material parameters are considered to be same as that for bulk germanium and are adapted from a standard manual on the Monte Carlo simulations [23]. The electrons are injected from the source with initial polarization in the $z$-direction (along the thickness of the wire). A time step of $0.02 \mathrm{fs}$ was chosen, and electrons were run for $1 \times 10^{6}$ such time steps to ensure that steady state has been reached. Data is recorded for the last 50,000 steps only. The ensemble average is calculated for each component of the spin vector for the last 50,000 steps at each point of the wire.

\section{Results and Discussion}

3.1. Spin Relaxation Lengths at Room Temperature (300 K) for a Driving Electric Field of $1 \mathrm{kV} / \mathrm{cm}$. A spin transport study was done at room temperature $(300 \mathrm{~K})$ for both $2 \mathrm{D}$ channels and $1 \mathrm{D}$ nanowires at a moderate driving electric field of $1 \mathrm{kV} / \mathrm{cm}$. Since the initial polarization is along the thickness of the wire, that is, in the $z$-direction, the ensemble averaged $x$ and $y$ components of spin fluctuate around zero (with very small magnitudes) and only the ensemble averaged $z$ component of spin decays along the length. Figures 2 and 3 show how the magnitude of spin vector decays along the $2 \mathrm{D}$ and $1 \mathrm{D}$ channel.

Spin-dephasing length in a nanowire is found to be around $210 \mathrm{~nm}$ compared to around $12 \mathrm{~nm}$ in a 2D channel. Thus the spin-dephasing lengths for a nanowire are about 18 times larger than two-dimensional channels. This result bears conformity with similar studies conducted earlier by researchers where improvements in spin relaxation lengths in $1 \mathrm{D}$ channels over $2 \mathrm{D}$ channels have been reported $[12,13$, 36, 37].

Explaining this difference in terms of difference in scattering rates in between nanowires and $2 \mathrm{D}$ channels meets with failure owing to the fact that mobility in nanowires has been found to be smaller than that in a $2 \mathrm{D}$ channel. The origin of this difference is due to the fact that the dominant

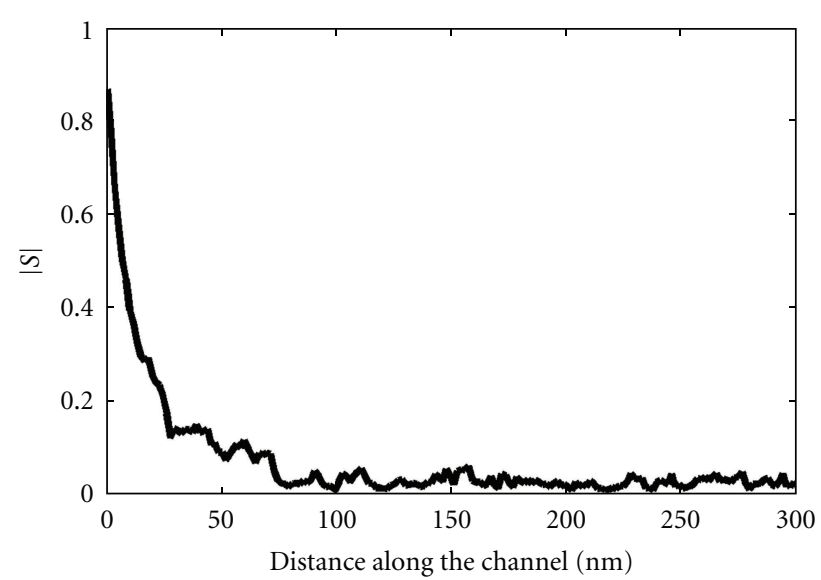

FIGURE 3: Variation of magnitude of spin along a 2D germanium channel.

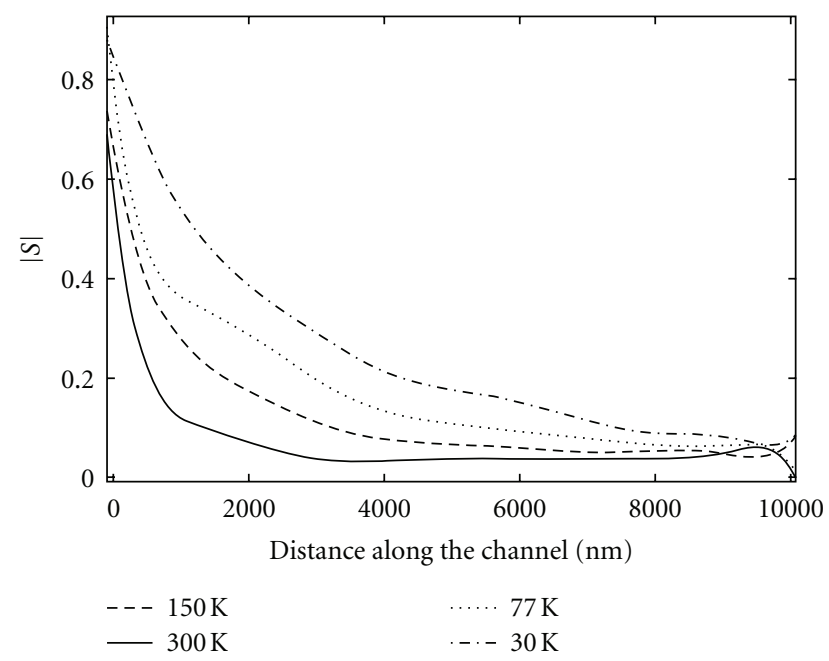

FIgURE 4: Variation of magnitude of spin along the channel for germanium nanowire at different temperatures at a driving electric field of $1 \mathrm{kV} / \mathrm{cm}$.

spin-relaxing mechanism, DP relaxation, is suppressed by confinement $[38,39]$. Thus a nanowire has considerably lesser DP relaxation than a 2D channel leading to larger dephasing lengths. A detailed explanation to this effect is reported in our previous work [25]. It must be mentioned that our results are consistent with the expectation that spin dephasing increases with the randomness of the motion. In a 2D channel, randomization of the electron motion occurs along two directions while in a nanowire it happens only in one direction.

3.2. Effect of Temperature. Figures 4 and 5 show the decay of the average spin vector at different temperatures for a nanowire and for a 2D channel, respectively, at a driving electric field of $1 \mathrm{kV} / \mathrm{cm}$. For a nanowire spin relaxation length increases from $210 \mathrm{~nm}$ at $300 \mathrm{~K}$ to $575 \mathrm{~nm}$ at $150 \mathrm{~K}$, to $940 \mathrm{~nm}$ at $77 \mathrm{~K}$, and $2.19 \mu \mathrm{m}$ at $30 \mathrm{~K}$. For a $2 \mathrm{D}$ channel relaxation length increases from $12 \mathrm{~nm}$ at $300 \mathrm{~K}$ to $30 \mathrm{~nm}$ at 


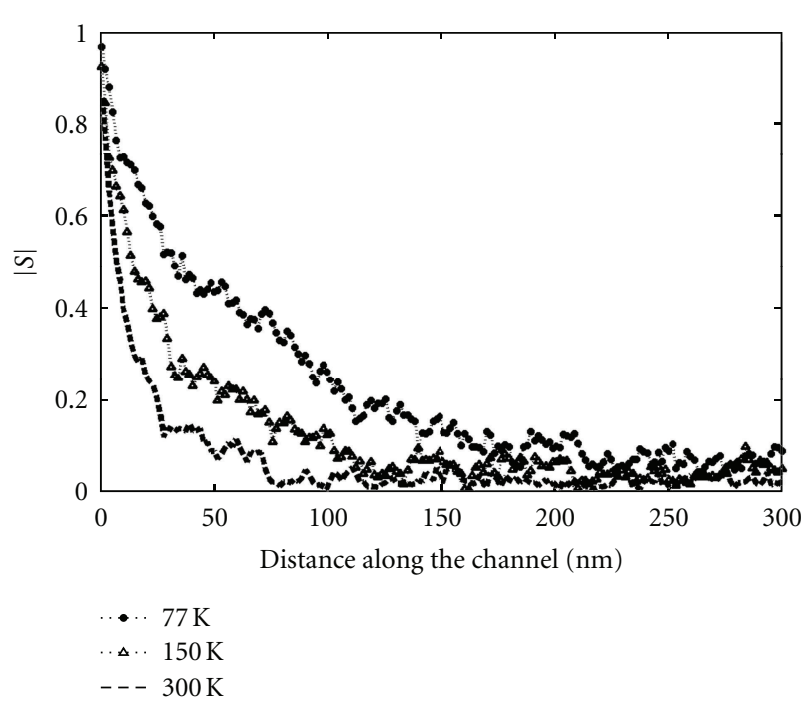

Figure 5: Variation of magnitude of spin along a 2D germanium channel at different temperatures at a driving electric field of $1 \mathrm{kV} / \mathrm{cm}$.

$150 \mathrm{~K}$ and to $70 \mathrm{~nm}$ at $77 \mathrm{~K}$. Thus spin relaxation length is observed to be a strong function of temperature, and thus information remains preserved in the spin of electrons up to a greater distance.

On increasing the crystal temperature, the phonon scattering rates increase. These increased scattering rates cause the electron to undergo scattering after very short time intervals and thus very short distances. This in turn randomizes the $k$ vector rapidly, and hence the precession vector also gets randomized within short distances from the source. Thus they get depolarized within smaller distances. This is again consistent with the fact that the more random the motion of electron (due to increased temperatures), the stronger the depolarization.

3.3. Effect of Applied Electric Field. Figures 6 and 7 show the decay of the magnitude of ensemble averaged spin vector at different driving electric fields for a nanowire and for a $2 \mathrm{D}$ channel at room temperature. The values of electric field used for analysis in our simulations are moderate enough to ensure that drift velocity saturation does not occur. The spin relaxation length for a nanowire changes from $193 \mathrm{~nm}$ at $100 \mathrm{~V} / \mathrm{cm}$ to $195 \mathrm{~nm}$ at $500 \mathrm{~V} / \mathrm{cm}$, to $210 \mathrm{~nm}$ at $1 \mathrm{kV} / \mathrm{cm}$, to $185 \mathrm{~nm}$ at $2 \mathrm{kV} / \mathrm{cm}$, and to $222 \mathrm{~nm}$ at $5 \mathrm{kV} / \mathrm{cm}$. The spin relaxation length for a $2 \mathrm{D}$ channel, however, shows a slight variation with applied electric field. The spin relaxation length for a $2 \mathrm{D}$ channel first decreases from $16 \mathrm{~nm}$ at $100 \mathrm{~V} / \mathrm{cm}$ to $13 \mathrm{~nm}$ at $500 \mathrm{~V} / \mathrm{cm}$ and to $12 \mathrm{~nm}$ at $1 \mathrm{kV} / \mathrm{cm}$. Thereafter it increases to $13 \mathrm{~nm}$ at $2 \mathrm{kV} / \mathrm{cm}$ and to $18 \mathrm{~nm}$ at $5 \mathrm{kV} / \mathrm{cm}$. As compared to the dependence of spin dephasing on temperature, the dependence of spin dephasing on driving electric field is found to be weak and clearly nonmonotonic. This is because it is manipulated by two opposing factors, ensemble averaged drift velocity and scattering rates.

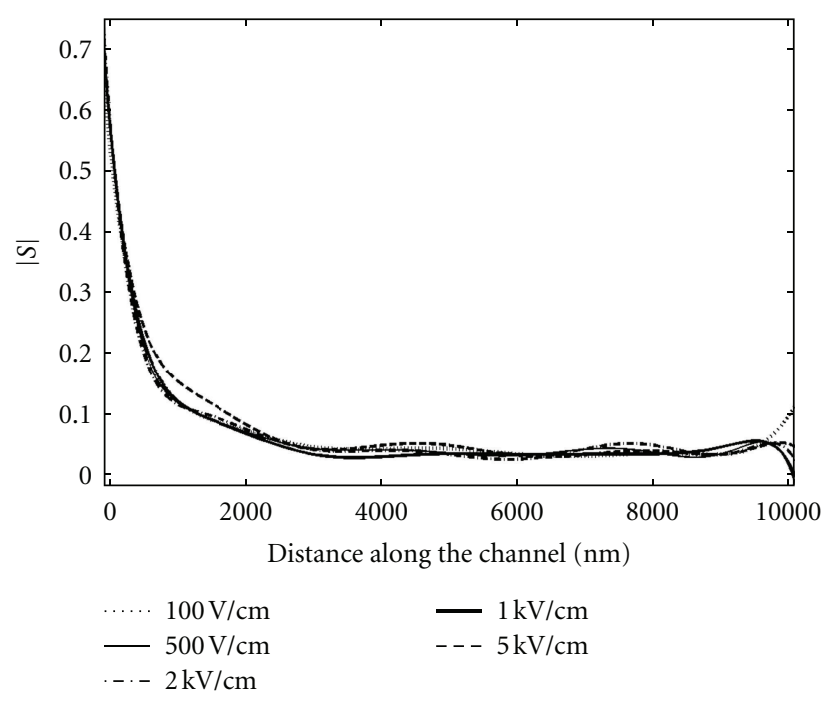

FiguRE 6: Variation of magnitude of spin along the channel for germanium nanowire at different driving electric fields at a temperature of $300 \mathrm{~K}$.

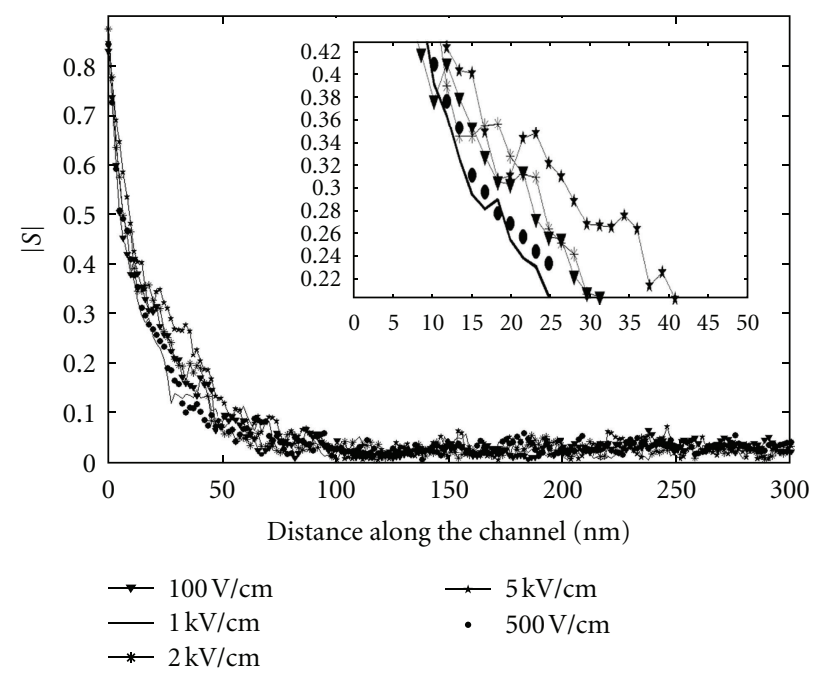

FIGURE 7: Variation of magnitude of spin along 2D germanium channel at different driving electric fields at a temperature of $300 \mathrm{~K}$.

Any dominance of drift velocity over the scattering rates makes the electron and hence the spin penetrates further into the channel leading to larger spin relaxation lengths. The reverse happens when scattering rates dominate over drift velocity and the increased scattering rates dephase the spin faster. The overall effect is decided by the dominant effect amongst the two.

In our four subband model, the intersubband scattering saturates after a point as we increase the driving electric field. At higher driving electric fields, the scattering rates remain fairly constant with only a slight variation. The drift velocity starts to dominate the scattering rates in this regime, and spin relaxation length starts to increase. This explains the increase in relaxation length at higher electric fields. Comparing with our work on spin transport in Si in [25], we note that Si has 
higher spin relaxation length than Ge. At a temperature of $300 \mathrm{~K}$ and an electric field of $1 \mathrm{kV} / \mathrm{cm}$, the spin relaxation length of Si for a 2D channel is $125 \mathrm{~nm}$ as opposed to $12 \mathrm{~nm}$ for Ge. The values for a 1D nanowire are $1.98 \mu \mathrm{m}$ for $\mathrm{Si}$ as opposed to $210 \mathrm{~nm}$ for Ge. The spin relaxation lengths for both $\mathrm{Si}$ and Ge vary with temperature and electric field.

\section{Conclusion}

In our work we show that confining the motion to only one direction can improve drastically upon the spin relaxation length (more than an order of magnitude to around $210 \mathrm{~nm}$ for a nanowire compared to $12 \mathrm{~nm}$ for a $2 \mathrm{D}$ channel at $300 \mathrm{~K}$ and driving electric field of $1 \mathrm{kV} / \mathrm{cm}$ ). Thus the information stored in the spin of electrons remains polarized up to a larger length on using nanowires due to suppression of DP relaxation. This larger spin relaxation length leads us to believe that spintronic devices can be efficiently implemented with nanowires. Also we observe that the spin relaxation length in nanowires can be further increased by reducing the temperature, in which case it increases to $940 \mathrm{~nm}$ at $77 \mathrm{~K}$ and $2.19 \mu \mathrm{m}$ at $30 \mathrm{~K}$. Thus lowering the working temperatures can improve the performance of spintronic devices manifold.

\section{References}

[1] A. Fert, "The present and the future of spintronics," Thin Solid Films, vol. 517, no. 1, pp. 2-5, 2008.

[2] I. Žutić, J. Fabian, and S. D. Sarma, "Spintronics: fundamentals and applications," Reviews of Modern Physics, vol. 76, no. 2, pp. 323-410, 2004.

[3] S. Bandyopadhyay and M. Cahay, Introduction to Spintronics, CRC Press, 2008.

[4] J. Fabian, A. Matos-Abiague, C. Ertler, P. Stano, and I. Žutić, "Semiconductor spintronics," Acta Physica Slovaca, vol. 57, no. 4-5, pp. 565-907, 2007.

[5] M. W. Wu, J. H. Jiang, and M. Q. Weng, "Spin dynamics in semiconductors," Physics Reports, vol. 493, no. 2-4, pp. 61236, 2010.

[6] A. Kamra, B. Ghosh, and T. K. Ghosh, "Spin relaxation due to electron-electron magnetic interaction in high Lande $\mathrm{g}$-factor semiconductors," Journal of Applied Physics, vol. 108, no. 5, Article ID 054505, 2010.

[7] D. Hägele, M. Oestreich, W. W. Rühle, N. Nestle, and K. Eberl, "Spin transport in GaAs," Applied Physics Letters, vol. 73, no. 11, pp. 1580-1582, 1998.

[8] L. Kong, G. Du, Y. Wang, J. Kang, R. Han, and X. Liu, "Simulation of spin-polarized transport in GaAs/GaAlAs quantum well considering intersubband scattering by the Monte Carlo method," in Proceedings of the International Conference on Simulation of Semiconductor Processes and Devices (SISPAD '05), pp. 175-178, September 2005.

[9] D. Sánchez, C. Gould, G. Schmidt, and L. W. Molenkamp, "Spin-polarized transport in II-VI magnetic resonanttunneling devices," IEEE Transactions on Electron Devices, vol. 54, no. 5, pp. 984-990, 2007.

[10] C. Tahan and R. Joynt, "Rashba spin-orbit coupling and spin relaxation in silicon quantum wells," Physical Review B, vol. 71, no. 7, pp. 1-7, 2005.
[11] S. P. Dash, S. Sharma, R. S. Patel, M. P. De Jong, and R. Jansen, "Electrical creation of spin polarization in silicon at room temperature," Nature, vol. 462, no. 7272, pp. 491-494, 2009.

[12] S. Pramanik, S. Bandyopadhyay, and M. Cahay, "Spin transport in nanowires," in Proceedings of the 3rd IEEE Conference on Nanotechnology, vol. 2, pp. 87-90, 2003.

[13] A. Bournel, P. Dollfus, P. Bruno, and P. Hesto, "Spin polarized transport in 1D and 2D semiconductor heterostructures," Materials Science Forum, vol. 297-298, pp. 205-212, 1999.

[14] P. Logan and X. Peng, "Strain-modulated electronic properties of Ge nanowires: a first-principles study," Physical Review B, vol. 80, no. 11, Article ID 115322, 2009.

[15] S. Patibandla, S. Pramanik, S. Bandyopadhyay, and G. C. Tepper, "Spin relaxation in a germanium nanowire," Journal of Applied Physics, vol. 100, no. 4, Article ID 044303, 2006.

[16] E. S. Liu, J. Nah, K. M. Varahramyan, and E. Tutuc, "Lateral spin injection and large spin diffusion length in germanium," Nano Letters, vol. 10, no. 9, pp. 3297-3302, 2010.

[17] S. Patibandla, G. M. Atkinson, S. Bandyopadhyay, and G. C. Tepper, “Competing D'yakonov-Perel' and Elliott-Yafet spin relaxation in germanium," Physica E, vol. 42, no. 5, pp. 17211726, 2010.

[18] M. I. D’yakonov and V. I. Perel, "Spin relaxation of conduction electrons in noncentrosymmetric semiconductors," Soviet Physics, vol. 13, no. 12, pp. 3023-3026, 1972.

[19] G. L. Bir, A. G. Aronov, and G. E. Pikus, "Spin relaxation of electrons scattered by holes," Soviet Physics, vol. 42, pp. 705712, 1976.

[20] R. J. Elliott, "Theory of the effect of spin-Orbit coupling on magnetic resonance in some semiconductors," Physical Review, vol. 96, no. 2, pp. 266-279, 1954.

[21] A. Martinez, R. Brown, and A. Asenov, "Full-band NEGF simulations of surface roughness in Si nanowires," Journal of Physics, vol. 242, Article ID 012016, 2010.

[22] C. Jacoboni and L. Reggiani, "The Monte Carlo method for the solution of charge transport in semiconductors with applications to covalent materials," Reviews of Modern Physics, vol. 55 , no. 3, pp. 645-705, 1983.

[23] C. Jacoboni and P. Lugli, The Monte Carlo Method for Semiconductor Device Simulation, Springer, Vienna, Austria, 1989.

[24] S. Saikin, M. Shen, M. C. Cheng, and V. Privman, "Semiclassical Monte Carlo model for in-plane transport of spinpolarized electrons in III-V heterostructures," Journal of Applied Physics, vol. 94, no. 3, pp. 1769-1775, 2003.

[25] A. Kumar, M. W. Akram, S. G. Dinda, and B. Ghosh, "Spin relaxation in silicon nanowires," Journal of Computational and Theoretical Nanoscience, In press.

[26] E. A. De Andrada E Silva, G. C. La Rocca, and F. Bassani, "Spin-split subbands and magneto-oscillations in III-V asymmetric heterostructures," Physical Review B, vol. 50, no. 12, pp. 8523-8533, 1994.

[27] W. Fawcett and E. G. S. Paige, "Negative differential mobility of electrons in germanium: a Monte Carlo calculation of the distribution function, drift velocity and carrier population in the (111) and (100) minima," Journal of Physics C, vol. 4, no. 13, pp. 1801-1821, 1971.

[28] P. H. Song and K. W. Kim, "Spin relaxation of conduction electrons in bulk III-V semiconductors," Physical Review B, vol. 66, no. 3, Article ID 035207, pp. 352071-352078, 2002.

[29] E. B. Ramayya, D. Vasileska, S. M. Goodnick, and I. Knezevic, "Electron transport in silicon nanowires: the role of acoustic 
phonon confinement and surface roughness scattering," Journal of Applied Physics, vol. 104, no. 6, Article ID 063711, 2008.

[30] J. Lee and H. N. Spector, "Impurity-limited mobility of semiconducting thin wire," Journal of Applied Physics, vol. 54, no. 7, pp. 3921-3925, 1983.

[31] R. Kotlyar, B. Obradovic, P. Matagne, M. Stettler, and M. D. Giles, "Assessment of room-temperature phonon-limited mobility in gated silicon nanowires," Applied Physics Letters, vol. 84, no. 25, pp. 5270-5272, 2004.

[32] P. J. Price, "Two-dimensional electron transport in semiconductor layers. I. Phonon scattering," Annals of Physics, vol. 133, no. 2, pp. 217-239, 1981.

[33] K. Yokoyama and K. Hess, "Monte carlo study of electronic transport in $\mathrm{Al}_{1-x} \mathrm{Ga}_{x} \mathrm{As} / \mathrm{GaAs}$ single-well heterostructures," Physical Review B, vol. 33, no. 8, pp. 5595-5606, 1986.

[34] H. Tanimoto, N. Yasuda, K. Taniguchi, and C. Hamaguchi, "Monte Carlo study of hot electron transport in quantum wells," Japanese Journal of Applied Physics, vol. 27, no. 4, pp. 563-571, 1988.

[35] V. M. Polyakov and F. Schwierz, "Monte Carlo calculation of two-dimensional electron gas mobility in InN-based heterostructures," Journal of Applied Physics, vol. 101, no. 3, Article ID 033703, 2007.

[36] S. Pramanik, S. Bandyopadhyay, and M. Cahay, "Decay of spin-polarized hot carrier current in a quasi-one-dimensional spin-valve structure," Applied Physics Letters, vol. 84, no. 2, pp. 266-268, 2004.

[37] Y. Kunihashi, M. Kohda, and J. Nitta, "Enhancement of spin lifetime in gate-Fitted InGaAs Narrow wires," Physical Review Letters, vol. 102, no. 22, Article ID 226601, 2009.

[38] A. W. Holleitner, V. Sih, R. C. Myers, A. C. Gossard, and D. D. Awschalom, "Dimensionally constrained D'yakonov-Perel' spin relaxation in $\mathrm{n}$-InGaAs channels: transition from $2 \mathrm{D}$ to 1D," New Journal of Physics, vol. 9, article 342, 2007.

[39] A. G. Mal'shukov and K. A. Chao, "Waveguide diffusion modes and slowdown of D'yakonov-Perel' spin relaxation in narrow two-dimensional semiconductor channels," Physical Review B, vol. 61, no. 4, pp. R2413-R2416, 2000. 

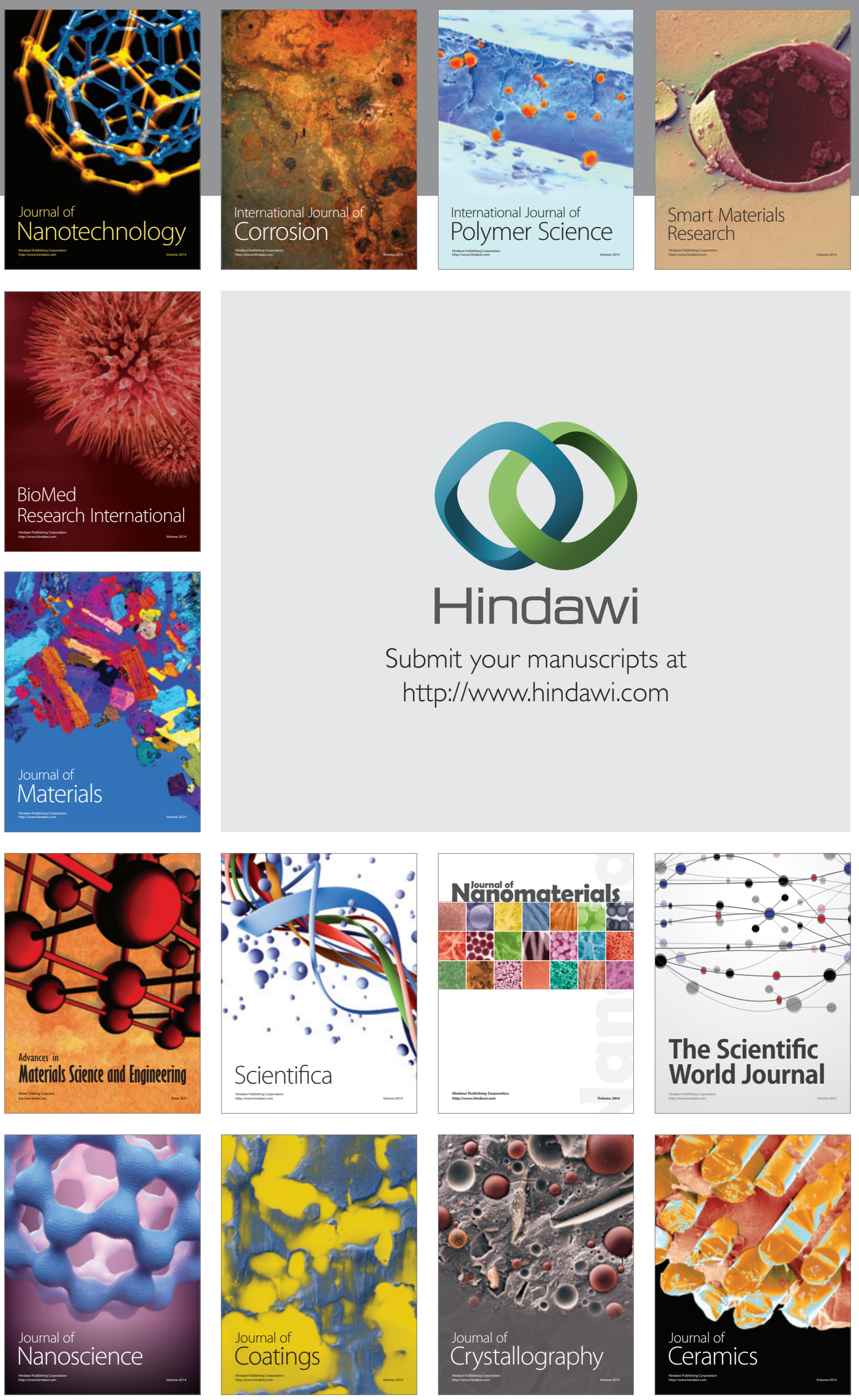

The Scientific World Journal

Submit your manuscripts at

http://www.hindawi.com

\section{World Journal}

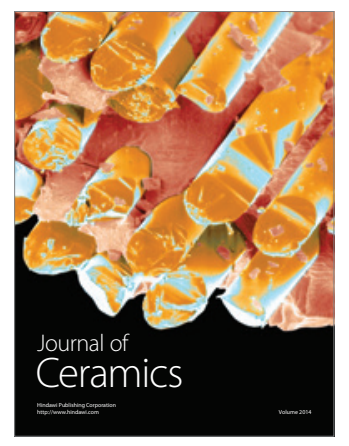

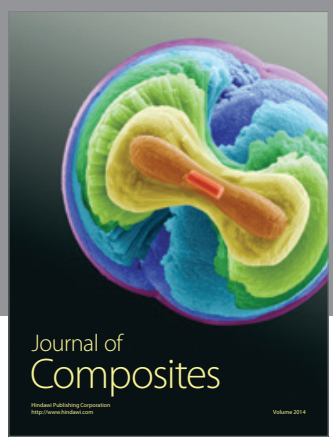
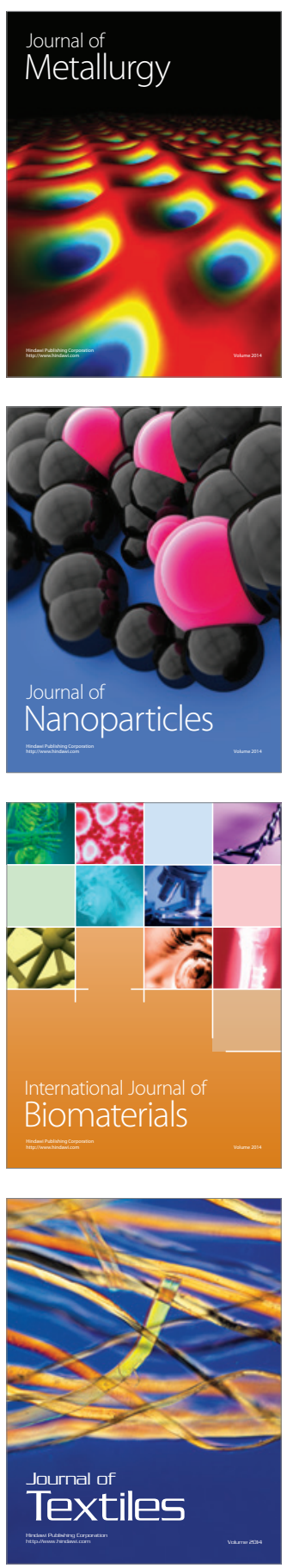